\title{
Design of Customized Fractal FSS
}

Anuradha, A.Patnaik, S.N Sinha

Department of Electronics and Computer Engineering

Indian Institute of Technology, Roorkee

Roorkee - 247667, India

anuradhasonker@yahoo.co.in, apatnaik@ieee.org,

sn_sinha@ieee.org

\begin{abstract}
A Novel design approach is proposed for designing customized fractal frequency selective surfaces (FSS) by combining the merits of Artificial Neural Network (ANN) and Particle Swarm Optimization (PSO). The trained ANN developed for analysis of fractal FSS is embedded in the optimization loop of PSO to find out the design dimensions of the unit cell of fractal FSS for user-defined stop band frequencies. The developed methodology is tested with Sierpinski carpet as the candidate structure and the effectiveness of the developed approach is confirmed by the simulation result.
\end{abstract}

\section{INTRODUCTION}

A FSS is a planar periodic structure in which radiation is either allowed to transmit through or blocked, depending upon the frequency of the incident wave, incident angle and polarization [1]. These surfaces have many applications in military as well as commercial sectors. One of the possible ways of creating multiband FSS is by using the fractal concept in designing structures. Several iterations of fractal can be used to design FSS that has a multiband frequency response.

Although number of analysis technique for FSSs have been reported in the literatures [2], but in design front, the literatures is limited [3]. Design of multi-frequency customized FSSs is a difficult task because it involves the optimization of number of variables. In this work, we have developed a user friendly module for design of fractal FSS at user defined frequencies. Although the same methodology can be extended for other fractal structures.

\section{SIERPINSKI CARPET FSS DESIGN}

The Sierpinski carpet as a FSS has been explored as an excellent candidate for multiband applications [4]. The classical structure of this FSS is shown in Figure 1.1.The scaling in this classical structure in both the directions is 0.5 . The centre patch corresponds to the lower frequency and the sub patches which are quarter size of the pervious patch correspond to the next higher frequencies. The size of centre patch $(L)$ and the scaling can be change to shift the operating frequencies of the FSS. The present work deals with the determination of this design parameters for the FSS to have a specific user defined frequency response.

Because most of the multi-frequency FSS are dual band or at the most triple band type, so in this work the design of a third

\author{
Juan R.Mosig \\ Laboratory of Electromagnetics and Acoustics (LEMA- \\ ELB) \\ EPFL - Station 11, CH-1015, Lausanne \\ Switzerland \\ juan.mosig@epfl.ch
}

iterated carpet is demonstrated for it to act as a multi bandstop screen. An iterated Function System for design of this carpet is discussed in [4]. In order to verify the frequency response of the FSS, the structure was simulated using the transient solver of CST microwave studio [5] for a normal incident plane wave. As the plane wave is x-polarized, so scaling $r_{\mathrm{x}}$ and $r_{1}$ (Figure 1.1) in $x$ directions of first and second iterated patch of FSS shows the effective transmission and reflections of the wave. The cell has periodicity $\mathrm{T}_{\mathrm{x}}$ and $\mathrm{T}_{\mathrm{y}}$ in $\mathrm{x}$ and $\mathrm{y}$ directions, and the size of the substrate on which the fractal structure is printed is determined by the periodicity $\mathrm{T}_{\mathrm{x}}$ and $\mathrm{T}_{\mathrm{y}}$.

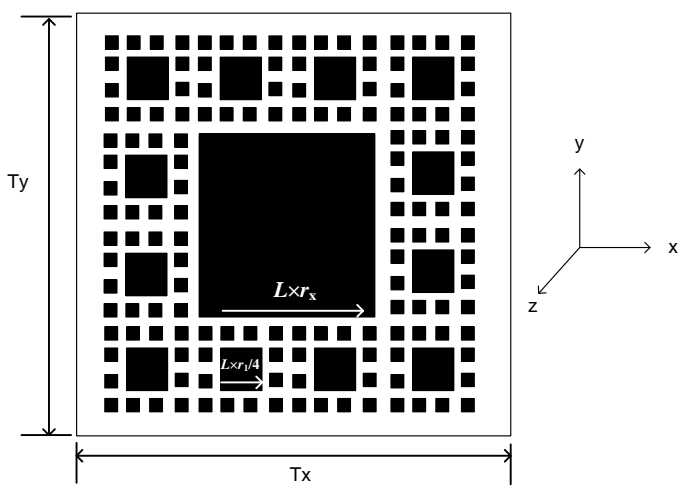

(Dimensions of First iterated square patch $=L \times r_{\mathrm{x}}$, Second iterated square Patches $=\left(L \times r_{1}\right) / 4$, Third Iterated square patches $\left.=\left(L \times r_{1}\right) / 4^{2}\right)$

Figure 1.1 A Modified Third Iterated Sierpinski Carpet FSSs unit cell structure

\section{PROBLEM FORMULATION}

In this paper the benefits of ANN and PSO are utilized to find out the optimized design parameters for the FSS.

The role of ANN is to form a mapping between the design parameters with the corresponding working frequencies (Figure 1.2). For ANN training, a set of self-similar Sierpinski carpet were formed by varying the scaling. The length $L$ of the carpet was varied between $10 \mathrm{~mm}$. - $120 \mathrm{~mm}$. in steps of $5 \mathrm{~mm}$. and the scaling $r_{\mathrm{x}}$ and $r_{1}$ was between $0.1-0.5$. Simulations were carried out to find out the operational frequencies of the third iterated carpet FSS. A total of 525 data sets of were created for the training of ANN. Here it may be noted that the 
properties of the dielectric constant 2.2 and thickness on which the FSS is printed was not taken as variable for data generation of ANN. A multilayer perceptron of size $3 \times 15 \times 3$ was trained in the backpropagation mode to develop the analysis model for the FSS. For ease of training the ANN output data were preprocessed to $f_{\mathrm{r} 1}, f_{\mathrm{r} 2} / f_{\mathrm{r} 1}$ and $f_{\mathrm{r} 3} / f_{\mathrm{r} 1}$ instead of the absolute value of the frequencies.

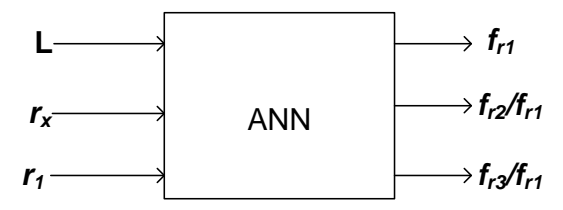

Figure 1.2 Sierpinski Carpet FSSs developed ANN Model

PSO was used as an optimizer to find the optimized unit cell dimensions of fractal FSS. The previously trained ANN was used to evaluate the cost function for the PSO. The particles positions and velocities were changed according to the following two equations [6].

$$
\begin{gathered}
v_{i d}=w v_{i d}+c_{1} \operatorname{rand}()\left(p_{i d}-x_{i d}\right)+c_{2} \operatorname{rand}()\left(p_{g i}-x_{i d}\right) \\
x_{i d}=x_{i d}+v_{i d}
\end{gathered}
$$

In the present paper the PSO was tuned by setting a small population size of ten, $c_{1}$ and $c_{2}$ accelerations terms, were set to 2.0 and the inertial weight $w$ was varied linearly from 0.9 to 0.4 over iteration, finally $w$ was set at 0.7 .

\section{RESULTS AND DISCUSSION}

The developed methodology was used to design number of FSSs at desired frequencies. A typical structure with bandstop frequencies at 2.4 and $5.2 \mathrm{GHz}$ is presented in this paper. The following cost function was used.

$$
\text { Fitness function }=\left(2.4-f_{r 1}\right)^{2}+\left(5.2-f_{r 2}\right)^{2}
$$

The final optimized dimensions of the FSS unit cell are given in Table I. The periodicity $\mathrm{T}_{\mathrm{x}}=201 \mathrm{~mm}$ and $\mathrm{T}_{\mathrm{y}}=201 \mathrm{~mm}$ in $\mathrm{x}$ and y directions.

The computation time were noted to be 15 second. These optimized dimensions were used to simulate the structure using CST. The frequency performance of the unit cell is shown in Figure 1.3. From the Figure the stopband behaviour of the FSS at 2.4 and $5.2 \mathrm{GHz}$ can be clearly marked.

\begin{tabular}{|c|c|c|c|c|c|}
\hline \multicolumn{3}{|c|}{$\begin{array}{c}\text { Optimized parameters } \\
\text { of FSS unit cell }\end{array}$} & \multicolumn{3}{|c|}{$\begin{array}{l}\text { The area of the each square patches of } \\
\text { each iteration of FSS }\end{array}$} \\
\hline$L(\mathrm{~mm})$ & $r_{x}$ & $r_{1}$ & $\begin{array}{l}\text { First } \\
\text { patch } \\
=L \times r_{x} \\
\left(\mathrm{~mm}^{2}\right)\end{array}$ & $\begin{array}{c}\text { Second } \\
\text { Patches }= \\
\left(L \times r_{1}\right) / 4 \\
\left(\left(\mathrm{~mm}^{2}\right)\right.\end{array}$ & $\begin{array}{c}\text { Third } \\
\text { patches }=\left(L \times r_{1}\right) / 4^{2} \\
\left(\mathrm{~mm}^{2}\right)\end{array}$ \\
\hline 200 & 0.3 & 0.5 & 60 & 25 & 6.25 \\
\hline
\end{tabular}

\section{TABLE I. OPTIMIZED DIMENSIONS OF THE FRACTAL} FSS.

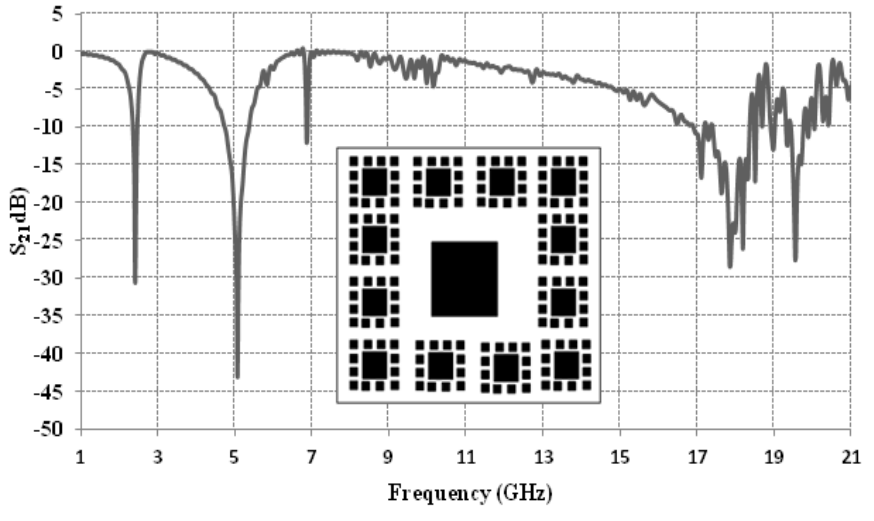

Figure 1.3 Transmission characteristics $\left(\mathrm{S}_{21}\right)$ at ISM band at 2.4, $5.2 \mathrm{GHz}$ and $\mathrm{mm}$ band at $17.88 \mathrm{GHz}$ over the range of 17$19 \mathrm{GHz}$ for optimized third Iterated Carpet FSS Structure for bandstop filter.

\section{CONCLUSION}

A novel design methodology has been proposed for design of customized FSSs. Although developed for Sierpinski carpet, the same methodology can be extended for design of other multiband fractal FSS.

\section{REFERENCES}

[1] Ben A. Munk, Frequency Selective Surfaces: Theory and Design, 1st ed. John Wiley \& Sons, New York, 2000.

[2] J. P. Gianvittorio, J. Romeu, S. Blanch and Y. Rahmat-Samii, "SelfSimilar Prefractals Frequency Selective Surfaces for Multil-band and Dual-Polarized Applications," in IEEE Transactions on Antennas and Propagation, vol. 51,No.15, November 2003.

[3] Asim Egemen Yilmazi and Mustafa Kuzuoglu, "Design of the Square Loop Frequency Selective Surfaces with Particle Swarm Optimization via the Equivalent Circuit Model," Radioengineering, Vol. 18, No. 2, pp. 95-102, June 2009.

[4] J. Romeu and Y. Rahmat-Samii , "Fractal FSS: A Novel Dual-band Frequency Selective Surface," IEEE Transactions on Antennas Propagation, vol. 48, No.7, pp. 1097-1105, July 2000.

[5] C S T Microwave Studio, http://www.cst.com.

[6] J.Robinson and Y.Rahmat-Samii, "Particle Swarm Optimization in Electromagnetic," IEEE Transactions on Antennas and Propagation, vol. 52, No. 2, pp. 397-407, Feburary 2004. 